\title{
Conflito de interesses na formação e prática do nutricionista: regulamentar é preciso
}

\author{
Conflict of interest in the training and practices of nutritionists: \\ regulation is necessary
}

Tatiane Nunes Pereira ${ }^{1}$

Fabiana Alves do Nascimento ${ }^{2}$

Daniel Henrique Bandoni ${ }^{3}$

${ }^{1}$ Programa de Pós-

Graduação em Saúde

Coletiva, Universidade

de Brasília. Campus

Universitário Darcy Ribeiro.

70910-900 Brasília DF

Brasil.

pereira.tnp@gmail.com

${ }^{2}$ Programa de Pós-

Graduação em Nutrição em

Saúde Pública, Universidade

de São Paulo. São Paulo SP

Brasil.

${ }^{3}$ Departamento de Saúde,

Clínica e Instituições,

Instituto de Saúde e

Sociedade da Universidade

Federal de São Paulo. São

Paulo SP Brasil.
Abstract Transnational "Big Food" companies use advertising strategies to influence nutritionists, professors and students of nutrition. There are, however, conflicts of interest in this relationship. The scope of this study is to conduct a narrative review on the influence of the food industry in training in nutrition. It was revealed that industries seek to induce the recommendation, the prescription and the consumption of products by students and nutritionists through strategies such as sponsorship of scientific meetings, travel funding and the distribution of promotional gifts. However, acceptance of these gifts can generate a moral obligation to reciprocate, thereby jeopardizing the judgment of information and decision on professional conduct. At the University, the advertising occurs mainly through sponsorship of events and research funding, donation of materials and structures and publicity in the classroom. Regulating the conduct of the private sector in the academic arena is essential and, in this perspective, the implementation of regulatory measures to limit the inclusion of the food industry in undergraduate courses in nutrition is recommended to ensure that nutritionists will be better prepared to perform their tasks in and ethical and unbiased manner.

Key words Food advertising, Food industry, Higher education, Conflict of interest, Nutrition in Public Health
Resumo As indústrias de alimentos utilizam estratégias de publicidade para influenciar nutricionistas, professores e estudantes de Nutrição, contudo, estas relações possuem conflitos de interesses. Este estudo objetiva realizar revisão narrativa sobre a influência das indústrias de alimentos na formação em Nutrição. Verificou-se que indústrias buscam induzir a recomendação, a prescrição e o consumo dos produtos por estudantes e nutricionistas, por meio de estratégias, como patrocínio de eventos científicos, financiamento de viagens e distribuição de brindes. Porém, aceitar a hospitalidade pode gerar obrigação moral de retribuir, comprometendo o julgamento das informações e a decisão sobre a conduta profissional. $\mathrm{Na}$ Universidade, a publicidade ocorre, principalmente, por meio do patrocínio de eventos e financiamento de pesquisas, materiais e estruturas e propaganda em sala de aula. Regulamentar a presença do setor privado na área acadêmica é fundamental e, nesta perspectiva, sugere-se a elaboração de medidas regulatórias que limitem a inserção de indústrias de alimentos nos cursos de graduação em Nutrição para que nutricionistas sejam mais bem preparados para desempenhar suas atribuições com ética e autonomia.

Palavras-chave Publicidade de alimentos, Indústria de alimentos, Educação superior, Conflito de interesses, Nutrição em Saúde Pública 


\section{Introdução}

A indústria moderna de alimentos surgiu após a Revolução Industrial e ganhou força no início do século XX, com a evolução de diversas etapas e novas formas de processamento ${ }^{1,2}$. Estas transformações também possibilitaram a criação de alimentos ultraprocessados (AUP), duráveis, convenientes, ultrapalatáveis e muito rentáveis ${ }^{1,3}$, de forma a criar novas necessidades e atender às características relacionadas a práticas alimentares na contemporaneidade, como a falta de tempo para o preparo e consumo de alimentos, flexibilização de horários e individualização dos rituais alimentares ${ }^{4}$.

A globalização da economia permitiu que os AUP fossem elaborados por indústrias transnacionais e comercializados em escala mundial, com a utilização de estratégias de publicidade para ampliar a venda e, consequentemente, o lucro ${ }^{5,6}$. O aumento da produção e da oferta e o excesso de propaganda destes produtos, associado à conveniência e ao baixo custo, têm induzido seu consumo elevado e influenciado a crescente prevalência de obesidade e outras doenças crônicas $^{7-13}$. No Brasil, o cenário não é diferente: o consumo de AUP tem aumentado e de alimentos in natura ou minimamente processados, diminuído, refletindo em quase $60 \%$ dos adultos com excesso de peso e cerca de $1 / 5$ com obesidade ${ }^{10,14,15}$.

Os impactos da alimentação e da obesidade na saúde levaram as indústrias a investirem na produção de AUP com apelo de beneficiar a saúde ${ }^{16-22}$. Nesse sentido, os alimentos industrializados propagandeados como 'saudáveis' são estrategicamente mitificados em função de seu teor nutricional e atributos funcionais ${ }^{23}$. Em geral, apesar de esses produtos conterem algum ingrediente indesejável reduzido, possuem outros com as mesmas características que permanecem em quantidades elevadas, além de serem mais ca$\operatorname{ros}^{22}$. Estima-se que a venda de tais produtos tenha representado cerca de 212 bilhões de dólares em 2012 em todo o mundo ${ }^{24}$, o que mostra sua importância para o lucro dos fabricantes.

As indústrias de alimentos também procuram convencer os governos de que a fortificação de AUP, como bebidas açucaradas, é a solução mais efetiva para combater deficiências nutricionais e induzir os consumidores a pensarem que os efeitos prejudiciais de sua ingestão excessiva são anulados, desestimulando o consumo de alimentos que são fontes naturais de micronutrientes ${ }^{8,21-22}$.

Nesse contexto, é possível observar a crescente influência dessas indústrias nas escolhas alimentares, com estratégias que buscam conquistar apoio desde consumidores e profissionais de saúde a agentes públicos governamentais para aumentar a venda de seus produtos e barrar medidas regulatórias. As mesmas indústrias procuram influenciar também nutricionistas, professores e estudantes de Nutrição para induzir a recomendação de seus alimentos, em especial, daqueles propagandeados como 'saudáveis'.

A relação de profissionais da saúde, pesquisadores, docentes, organizações de saúde, órgãos governamentais e entidades de classes profissionais com indústrias de alimentos possui inerentes conflitos de interesses (CoI), caracterizados aqui como condições que comprometem, de maneira imprópria, interesses primários e coletivos em função de interesses secundários ${ }^{25,26}$. $\mathrm{Na}$ área da saúde, por exemplo, os interesses primários podem ser o bem-estar dos indivíduos, a integridade das pesquisas e a formação de estudantes de cursos da área, e os secundários, posições privilegiadas nas indústrias ${ }^{27}$ e financiamento de pesquisas e eventos científicos ${ }^{28}$.

$\mathrm{Na}$ literatura essa discussão tem sido voltada principalmente à relação de indústrias de alimentos e pesquisadores, entidades de classe e organização de congressos de Nutrição ${ }^{27,28}$. No entanto, não há estudos que discutam a existência do CoI em espaços de formação e as estratégias utilizadas por essas indústrias. Assim, o presente trabalho tem como objetivo realizar revisão narrativa sobre a influência das indústrias de alimentos na formação em Nutrição.

\section{Trajetória metodológica}

Trata-se de estudo exploratório, realizado por meio de revisão narrativa da literatura científica sobre a influência das indústrias de produtos alimentícios na formação e atuação dos nutricionistas. Tal revisão é a mais apropriada para descrever o desenvolvimento de problemas em que a pesquisa original é escassa, visto que permite uma busca mais abrangente de material bibliográfico, sendo fundamental para a ampliação da perspectiva sobre o tema ${ }^{29,30}$.

As bases de dados pesquisadas foram: PubMed, do National Center for Biotecnology Information; Literatura Latino-Americana e do Caribe em Ciências da Saúde; e Scientific Electronic Library Online.

No intuito de contemplar toda a produção científica disponível, o período de publicação não foi delimitado. Foram associados os descritores Nutrição ou Alimentação aos descritores 
Conflito de Interesses, Interesses ou Ética; Indústria de Alimentos, Indústria de Processamento de Alimentos ou Tecnologia, Indústria e Agricultura; Formação, Ensino, Professores, Educação, Graduação, Educação de Pós-Graduação ou Instituições Acadêmicas; e Financiamento de Congressos e Reuniões Científicas.

Uma vez que a relação entre indústria de alimentos e formação dos nutricionistas é pouco abordada em estudos originais, foram incluídos artigos de diferentes naturezas bibliográficas como editoriais, relatórios, resenhas, entre outros que avaliassem dados sobre o impacto de patrocínios de sociedades e eventos científicos na formação e atuação dos nutricionistas.

Foram analisados os resumos de 113 estudos previamente selecionados pelo título. Entre esses, 31 discutiam a temática e foram apresentados neste artigo. Complementarmente, foram adicionadas 12 publicações que trataram da influência da indústria farmacêutica na formação de outros profissionais da saúde, tendo em vista que utiliza estratégias de publicidade semelhantes às empregadas pelas indústrias de alimentos ${ }^{6}$ e esta discussão está mais consolidada em outras áreas, como a Medicina. Todas as publicações encontradas são datadas a partir de 1990, demonstrando que a preocupação com o tema é recente.

Para apresentação dos resultados e discussão da revisão, optou-se pela estrutura de três seções, organizadas de acordo com os assuntos mais discutidos nos trabalhos encontrados, de forma a contextualizar a relevância deste tema na área da Nutrição, culminando na construção de recomendações direcionadas a universidades, professores e estudantes acerca da relação entre indústrias e universidades.

\section{Conflitos de interesses em políticas de alimentação e nutrição}

As parcerias entre indústrias de alimentos e organizações de saúde e segurança alimentar e nutricional, órgãos governamentais e entidades de classe de Nutrição (como conselhos, associações e sindicatos) atribuem credibilidade às primeiras por relacionar a marca de instituições reconhecidas por defender a saúde pública aos produtos por elas desenvolvidos, ofuscando aspectos negativos relacionados ao consumo dos seus produtos e ajudando, assim, a fidelizar os consumidores $^{21,27}$. Além disso, tais atores acabam por fazer propaganda da indústria ${ }^{27}$, chancelando as suas ações e confundindo consumidores e profissionais.
No entanto, parcerias entre organizações de saúde e órgãos governamentais com indústrias específicas, cujos CoI são evidentes, são diferentes de parcerias com o setor produtivo organizado, em que a linha entre os benefícios para saúde da população e os potenciais CoI é mais tênue. Por exemplo, sabe-se que o teor de sódio em alimentos industrializados no Brasil aumentou na última década, o consumo diário deste nutriente está acima do recomendado e sua redução em AUP é uma das estratégias nacionais para agir sobre o problema, por meio de acordo entre governo e setor produtivo ${ }^{31,32}$. Nesse caso, é uma estratégia válida de proteção à saúde em uma agenda de redução de riscos, porém, é necessário que, em sua implementação, todas as etapas da parceria ocorram de forma transparente, com definição de metas que impactem na redução do nutriente e evite-se o uso da pactuação e desta redução como publicidade para promover a venda desses alimentos ${ }^{31}$.

Recentemente foi lançada publicação da Union of Concerned Scientists que discute as estratégias utilizadas pelas corporações transnacionais para corromper a ciência com a intenção de influenciar políticas públicas. Os autores mostram que a atuação das indústrias ocorre em todo o ciclo dessas políticas, passando por financiamento, manipulação e introdução de vieses em pesquisas científicas, intimidação e difamação de cientistas, realização de campanhas junto à sociedade, influência da mídia, financiamento de campanhas eleitorais e realização de lobbies junto ao poder público ${ }^{33}$.

Nesse sentido, no âmbito acadêmico, pesquisadores são pagos para conduzir estudos e publicar artigos científicos favoráveis aos produtos das indústrias financiadoras com intenção de conter as evidências prejudiciais à venda de seus produtos, confundir o público, dar argumentos aos políticos aliados e prevenir ações governamentais contrárias às suas ações. Além disso, é disponibilizado suporte financeiro, que cobre a participação gratuita de pesquisadores em conferências científicas, viagens, hotéis e refeições ${ }^{21,28,34-36}$.

Um exemplo nesse sentido é o das indústrias fabricantes de açúcar, que financiam pesquisas para manter a incerteza sobre os danos do uso deste produto e pressionam instâncias decisórias governamentais para garantir que as recomendações nacionais sejam vagas e evitar que qualquer proposta de regulação ou taxação seja aprovada ${ }^{37}$. Ainda pode ser citada a influência das indústrias de laticínios, por meio de sua relação com organizações públicas responsáveis pela elaboração 
de recomendações nutricionais, na definição de elevada recomendação nutricional relacionada ao consumo de leite e derivados, ignorando variações individuais nas necessidades destes alimentos e hábitos de grupos étnicos específicos ${ }^{38}$.

Reconhece-se que pesquisadores e universidades precisam de financiamento para pesquisas e auxílio para a realização de eventos científicos, porém, é necessário avaliar as consequências para a credibilidade dos resultados encontrados e do conteúdo apresentado nos eventos ${ }^{28,39}$. Há instituições públicas que patrocinam a organização de eventos, permitindo sua realização somente com o pagamento das inscrições e financiamento público e comprovando que parcerias "públicas-públicas" são suficientes para garantir a viabilidade de construção de eventos autônomos e livres de $\mathrm{CoI}^{40}$.

Em relação à intervenção mais direta das indústrias de alimentos nas etapas de elaboração e implementação de políticas públicas, serão citados exemplos recentes ocorridos no Brasil e nos Estados Unidos.

No Brasil, após a realização de processo de construção democrática, inclusive com a participação do Conselho Nacional de Autorregulamentação Publicitária - CONAR (organização de agências de publicidade, anunciantes e veículos de comunicação, que objetiva fiscalizar as propagandas veiculadas no Brasil), e de associações relacionadas a indústrias de alimentos, a Agência Nacional de Vigilância Sanitária publicou a Resolução n²4/2010, para regular a publicidade de alimentos ricos em açúcar, gordura saturada e trans e sódio e bebidas com baixo teor nutricional. Após a publicação, diversas ações judiciais contrárias à aplicação da Resolução, promovidas pelo próprio CONAR e associações de indústrias de alimentos que participaram do processo, resultaram na emissão de parecer da Advocacia Geral da União, alegando que a RDC não era competência da Anvisa, e em sua suspensão $0^{41,42}$.

Nos EUA, a prefeitura de Nova Iorque limitou o tamanho das porções de refrigerantes e outras bebidas açucaradas colocadas à venda. Contudo, indústrias de bebidas americanas investiram milhões em campanhas para a população, alegando que o governo estava infringindo a liberdade do consumidor, e entraram com processos judiciais contra o Estado. A iniciativa foi revogada pela Corte de Apelação do Estado, tendo vigorado por menos de dois anos ${ }^{43}$.

Esses exemplos mostram a ação de indústrias de alimentos na implementação de políticas, quando houve tentativa de intervenção estatal.
Contudo, é possível apresentar a atuação dessas indústrias em outras etapas do ciclo de políticas públicas. Nesse contexto, pode ser citada a presença das indústrias de alimentos no Congresso Americano, a qual impediu a aprovação da alteração de regras da alimentação escolar que valorizavam os alimentos in natura em detrimento de produtos processados ${ }^{44}$.

As indústrias também se transvestem de sociedade civil a fim de influenciar políticas públicas. Nos EUA foi criado o grupo Americans Against Food Taxes, descrito como coalização de cidadãos interessados no tema, contrários à taxação de produtos, como bebidas açucaradas. Yanamandala et al. ${ }^{45}$ fizeram levantamento dos membros dessa organização e verificaram que $73 \%$ (364) vendiam alimentos e bebidas ou forneciam produtos para as indústrias. Somente 30 , de 498 membros, eram organizações da sociedade civil, sendo que 28 recebiam patrocínio de indústrias ${ }^{45}$. Já no Brasil, pode ser destacada a atuação de indústrias farmacêuticas sobre associações de pacientes com a finalidade de estimulá-los a realizarem ações judiciais para fornecimento de insumos não disponibilizados pelo governo $\mathrm{e}$ que, muitas vezes, ainda não possuem registro pela Anvisa ${ }^{46}$.

As indústrias de alimentos são organizadas e possuem força política e econômica, contando com representação e influência no governo e agindo de forma agressiva na tentativa de moldar a opinião pública e frear a ação regulatória. Com os discursos de defesa da responsabilidade individual, em relação ao consumo excessivo de AUP e ao sedentarismo, e de que as regulações lesam a liberdade de expressão das indústrias, convencem gestores e profissionais da saúde a defenderem ações e programas de nutrição ineficazes ${ }^{21,47}$.

\section{O interesse das indústrias de alimentos nos espaços de formação e prática do nutricionista}

Além das estratégias discutidas no tópico anterior, outras são direcionadas a profissionais de saúde e estudantes com objetivo de influenciá-los na indicação de produtos, uma vez que a população confia nas informações obtidas desses atores para tomada de decisões favoráveis à saúde ${ }^{48,49}$.

Os nutricionistas são referências sobre atenção dietética e relações entre alimentação, nutrição, promoção da saúde e prevenção de doenças, possuindo importante papel como formadores de opinião para a população e demais profissionais da saúde. É sua responsabilidade compar- 
tilhar práticas e saberes com equipes de saúde e população e auxiliar no desenvolvimento de ações para a valorização de hábitos alimentares saudáveis, evitando uma postura dominadora e reconhecendo o saber do outro como fundamental para a construção do seu ${ }^{50-53}$.

Ainda, faz parte de suas atribuições: realizar ou supervisionar compras de produtos alimentícios em Unidades de Alimentação e Nutrição; estabelecer as especificações para a compra de fórmulas nutricionais; realizar orientação e prescrição dietética; desenvolver pesquisas e estimular permanentemente postura ética nos estudantes, por meio de ações e exemplos na docência ${ }^{54}$. Além disso, o nutricionista pode ser responsável pela análise técnica e definição de alimentos e produtos a serem adquiridos em compras institucionais e atuar na gestão das ações de alimentação e nutrição e em etapas da cadeia produtiva de alimentos. Desta forma, é essencial que sua atuação e tomada de decisões sejam autônomas, independentes e imparciais à influência de interesses privados e ocorram dentro dos mais altos padrões de qualidade e princípios éticos ${ }^{55}$, priorizando a saúde da população e não se deixando influenciar por eventuais benefícios recebidos.

Contudo, destaca-se que há diversos fatores envolvidos nas escolhas alimentares e a efetivação de uma alimentação adequada e saudável, que vão além da atuação do nutricionista ${ }^{56}$. Além disso, a formação básica desse profissional é focada em aspectos bioquímicos e prescritivos, com pouca abordagem sobre princípios éticos, sociais, econômicos e ambientais ${ }^{56,57}$, o que também pode ter implicação na pouca atuação e percepção sobre a complexa rede que o profissional está inserido.

A identificação do cenário de atuação e das lacunas na formação do nutricionista permite compreender a importância da publicidade das indústrias direcionada a esses profissionais, com objetivo de induzir a prescrição, a aquisição, a utilização e o consumo de alimentos. Abordar futuros nutricionistas já na graduação é uma das formas de publicidade e é sobre essa questão que iremos nos debruçar a seguir.

\section{Entidades de classe de Nutrição e eventos científicos}

Diversos são os espaços em que as indústrias de alimentos atuam para persuadir nutricionistas e estudantes de graduação em Nutrição. Sabe-se que estudantes têm as entidades de classe como referência para sua atuação e participam ativamente de eventos científicos promovidos por es- $\operatorname{sas}^{58,59}$, sendo esses ambientes oportunos para o investimento das indústrias de alimentos.

Relatório que discute a influência de indústrias de alimentos e bebidas sobre a Academy of Nutrition and Dietetics (AND) dos Estados Unidos apontou aumento de mais de três vezes, entre 2001 e 2011, no número de patrocinadores relacionados às indústrias, ao mesmo tempo em que verificou a criação de programas de parcerias que possibilitam aos parceiros o uso do logotipo da AND em seus produtos, acesso aos e-mails dos membros, promoção de cursos aos profissionais registrados, entre outras práticas que colocam em questão a credibilidade da $A N D$ e de seu posicionamento político frente ao governo e à sociedade ${ }^{58}$.

Em relação aos eventos científicos, o maior evento promovido pela AND contava com mais de 300 expositores e $23 \%$ dos palestrantes eram diretamente empregados pelas indústrias de alimentos ou possuíam vínculos atuais ou passados com as mesmas, sendo que o potencial CoI não estava descrito na programação $0^{58}$.

Outra questão relevante a ser destacada é que materiais científicos distribuídos pelas indústrias em eventos científicos têm predominância de citações de pesquisas selecionadas e/ou financiadas pelas corporações, apresentando resultados favoráveis aos seus produtos ${ }^{45,60}$.

Pesquisa realizada por Reitshamer et al. avaliou a opinião de três mil associados à $A N D$ sobre os patrocínios e concluiu que há impacto negativo sobre a percepção do público, sobre a profissão e a credibilidade pessoal dos associados, sendo que a maioria estaria disposta a pagar mais para a $A N D$ ter menos patrocinadores ${ }^{60}$.

Em relação aos brindes, estudos demonstram que aceitá-los pode gerar obrigação moral de retribuir o agrado, comprometer o julgamento sobre as informações recebidas e a decisão sobre as condutas $^{61,62}$. O valor dos brindes compõe os gastos com publicidade das empresas e o seu retorno se dá por meio do pagamento que os pacientes realizarão com a compra do produto indicado pelos profissionais influenciados ${ }^{63}$.

Trabalhos realizados com médicos demonstram que a maioria recebe representantes da indústria farmacêutica e aceita brindes, porém, nega que sua conduta possa ser influenciada. Estes profissionais não questionam a confiabilidade das informações fornecidas pelos representantes, mas acreditam que os brindes podem influenciar a prescrição de colegas ${ }^{61,64}$.

Nesse sentido, o relatório sobre a $A N D$ relata ainda que alguns associados não se importam 
com o patrocínio das indústrias alimentícias em eventos científicos, alegando ser imunes à sua influência, porém, o que se observa é que não há questionamento sobre a confiabilidade das informações transmitidas pelas mesmas ${ }^{58}$.

As apresentações realizadas pelas indústrias em eventos científicos permitem o envio de mensagens enviesadas aos participantes sobre promoção da saúde e de prevenção das doenças, expondo também os estudantes à influência destas empresas $^{58,60}$.

\section{O papel das universidades}

O assédio da indústria, além de atingir profissionais, que trabalham prestando diretamente os serviços de saúde, também possui a finalidade de criar vínculos com os professores e os estudantes em universidades ${ }^{21,26,35,65}$.

As universidades são o principal lócus de formação de profissionais da saúde, de produção e disseminação de conhecimento e de desenvolvimento de pesquisas que servem como base para a elaboração de políticas públicas com implicações em diversos setores da sociedade ${ }^{66}$. Além disso, são responsáveis pela formação de líderes políticos e sociais ${ }^{67}$.

Desta forma, a universidade configura-se como um ambiente oportuno para a inserção das indústrias ${ }^{68}$ e a promoção dos produtos ocorre, principalmente, por patrocínios de atividades estudantis, financiamento de pesquisas, equipamentos, estruturas e propaganda e distribuição de brindes.

Mesmo que os estudantes recebam materiais e brindes sem proposta de contrapartida, é formada associação positiva entre a indústria e o profissional ${ }^{63}$. O contato da indústria com os estudantes, vulnerabilizados pela sua inexperiência profissional e crença de que o ambiente acadêmico é neutro, visa moldar valores e atitudes ${ }^{69}$.

Wazana $^{70}$, ao analisar 16 estudos sobre a relação entre indústrias farmacêuticas e médicos, observa que a ligação que inicia durante a formação universitária é mantida após a graduação.

Estudo realizado no Canadá comparou práticas de residentes submetidos à exposição da indústria farmacêutica durante a formação com aqueles não expostos. Os resultados demostraram que os não expostos consideravam menos as informações de representantes comerciais em sua atuação, concluindo que restringir o acesso dos representantes da indústria a estudantes é benéfico para a prática profissional ${ }^{71}$. Rogers et al. ${ }^{72}$ afirmam que, sobre o ponto de vista ético, a me- lhor política para os estudantes é não ter contato com a indústria.

Nesse sentido, Kao et al. ${ }^{73}$ verificaram que estudantes de Medicina que receberam mais informações ao longo do curso sobre as estratégias de marketing utilizadas pelas indústrias e estudantes de faculdades com políticas restritivas tinham maior probabilidade de apoiar a proibição de todas as interações entre representantes farmacêuticos e estudantes de Medicina.

No tocante à relação entre professores e indústrias, pesquisa realizada nos Estados Unidos com 82 professores e 333 estudantes de Medicina Veterinária no estado do Colorado indicou que $85,4 \%$ dos docentes entrevistados recebiam visitas de representantes comerciais e todos aceitavam brindes. Mais da metade dos professores confiava nas informações recebidas pelas indústrias e $36 \%$ utilizavam-nas como fonte de conhecimento. Além disso, 70,8\% dos estudantes apontaram as informações obtidas dos professores como uma das principais influências para a prescrição ${ }^{74}$.

Se o ambiente de ensino deve garantir ao estudante formação independente baseada em princípios ético-humanísticos, que o prepare para acompanhar criticamente as mudanças científicas e tecnológicas ocorridas em sua área de atuação, qual deve ser o papel da universidade diante da crescente participação do setor privado na sociedade e da interferência, constatada na literatura, deste setor na formação dos profissionais?

A autonomia universitária é requisito fundamental para a criticidade do conhecimento produzido, pois possibilita análise independente das relações entre ciência e desenvolvimento tecnológico, compreendendo os diferentes aspectos e o contexto social em que a universidade está inserida, evitando interferência de interesses mercadológicos ou políticos ${ }^{68,71}$.

Silva $^{66}$ aponta que as universidades que alcançaram altos padrões de ensino e pesquisa foram aquelas que mantiveram independência às investidas do mercado, valorizando o desenvolvimento social e a emancipação dos sujeitos. Assim, estas não devem deixar de serem centros de pesquisas e de incentivo ao pensamento crítico para se tornarem lócus de atividade impulsionada pelas exigências mercadológicas ${ }^{68}$.

Aqui se faz necessário reconhecer diferenças na natureza das instituições públicas e privadas responsáveis pela formação do nutricionista. No bojo do processo de privatização do ensino superior iniciado na década de 90, a expansão dos 
cursos de Nutrição no país entre 1996 e 2008 foi de $620,93 \%$ concentrado no setor privado não universitário ${ }^{75}$. Em 2009, existiam 391 cursos de Nutrição no Brasil, sendo $83 \%$ destes privados ${ }^{76}$, seguindo a tendência dos demais cursos da área da saúde no país ${ }^{75}$. Entretanto, nenhum estudo que abordasse as possíveis diferenças na formação do nutricionista e o CoI nesses espaços foi encontrado.

As indústrias de alimentos não têm a finalidade de educar ou produzir ciência; seu interesse é o lucro gerado pela comercialização dos produtos. Quando enviam representantes ou contratam "especialistas” para tal fim, as mensagens transmitidas são alinhadas com os objetivos de venda. Sendo assim, apesar de as informações parecerem confiáveis e baseadas em evidências científicas, são tendenciosas e objetivam a divulgação da marca. Além disso, a influência e o controle exercido nas agendas educacionais reduzem o espaço para que estudantes e profissionais reflitam sobre questões que possam ir contra os interesses das corporações ${ }^{58}$.

Tais empresas também têm oferecido estágios para estudantes de Nutrição. Apesar da atuação nas indústrias ser legítima e descrita nas funções do nutricionista ${ }^{54}$, a captação de estudantes em estágios, na maioria das vezes, melhor remunerados e com mais auxílios, em comparação àqueles em órgãos públicos, tem objetivo de criar vínculo positivo e duradouro entre o futuro profissional e a indústria.

Nesse sentido, cabe inclusive questionar os CoI intrínsecos à atuação do nutricionista nas indústrias de alimentos. O nutricionista pode ser contratado por essas empresas para promover a melhoria do perfil nutricional dos alimentos, contudo, sua atuação é subordinada a decisões que não visam à promoção da saúde, mas à garantia de lucros para as empresas. Assim, é de responsabilidade das entidades de classe de Nutrição não só indagar em que situações é possível legitimar a atuação nesta área, como também criar regulamentações adequadas para o nutricionista e o estudante de nutrição.

No tocante ao vínculo de professores às indústrias de alimentos, por meio de consultorias ou recebimento de financiamentos para a realização de pesquisas, as informações que os estudantes utilizam como base teórica para a atuação podem ser enviesadas pelo próprio professor, como descrito a seguir.

MacDonald e Williams-Jones ${ }^{65}$ analisaram a relação entre estudantes e professores que receberam financiamento de indústrias para a reali- zação de pesquisas e seus laboratórios. O estudo discute que a relação entre professor e estudante possui CoI inerentes, principalmente quando o professor é seu orientador, em função da relação de poder existente. A supervisão exige definição do tipo e quantidade de trabalho desenvolvido pelo estudante ${ }^{65}$, que podem estar relacionadas às empresas financiadoras, com pré-definição de resultados favoráveis aos seus produtos ou omissão de resultados negativos; avaliação, relacionada ao recebimento de auxílio financeiro e a futuras pesquisas realizadas pelo estudante junto às instituições de financiamento, e elaboração de cartas de recomendação ${ }^{65}$, as quais podem ser influenciadas pela aceitação ou negativa do estudante em divulgar falsos resultados ou omitir os verdadeiros.

Os professores têm o dever de orientar os estudantes sobre como acessar conhecimentos independentes ${ }^{65}$ e realizar avaliação crítica sobre as informações que encontram ou recebem. A ligação dos professores com indústrias pode enviesar o aconselhamento sobre publicação de resultados não favoráveis a estas, a escolha sobre os temas de pesquisa e sobre a carreira que este estudante irá seguir ${ }^{65}$.

Em relação aos temas abordados em sala de aula, a declaração de CoI, tanto de professores quanto dos palestrantes poderia ser uma alternativa para garantir a transparência, porém, não é suficiente, pois faz com que o profissional sinta-se livre para adotar comportamentos antiéticos ${ }^{73-74}$, como promover os produtos alimentícios da indústria a que este tem vínculo. Assim, é necessário que as universidades tenham maior controle da presença de empresas privadas, que apresentam CoI com os cursos, e da relação destas com os professores e pesquisadores da instituição de ensino.

\section{Regulação da presença de indústrias de alimentos na formação em nutrição}

É fundamental proteger os estudantes das ações e estratégias de publicidade das indústrias transnacionais de alimentos. Apesar da crescente preocupação com a influência da indústria na formação dos estudantes, não há propostas de regulação ou discussão dos limites para o assédio aos estudantes.

Revisão bibliográfica norte-americana mostra que a maioria dos estudantes de Medicina é favorável à limitação da participação da indústria em sua formação, entretanto os motivos e a forma como isso pode ser feito diferem em cada estudo ${ }^{77}$. 
A Associação Australiana de Estudantes de Medicina elaborou um guia para determinar os princípios que governam as interações entre estudantes de medicina e empresas farmacêuticas ${ }^{78}$. Apesar de facultativo, o guia tenta estabelecer limites entre a relação da indústria com o ensino, reconhecendo que esta é grave questão ética que deve ser debatida.

Também existem exemplos de iniciativas governamentais que ajudam a minimizar a influência de interesses privados em pesquisas e formação dos estudantes. Em Washington (DC), foram estabelecidas regras sobre $\mathrm{CoI}$ em pesquisas, que afetam cerca de 40 mil pesquisadores e sua violação implica a suspenção de recursos federais para o financiamento de pesquisas. Da mesma forma, a Agência Europeia de Medicina criou um banco de dados que permite acesso a declarações de CoI de pesquisadores. A existência de declarações neste banco impede a participação em conselhos consultivos governamentais ${ }^{79}$.

Diversos autores discutem sobre como evitar os CoI existentes na relação de pesquisadores e docentes com a iniciativa privada, estabelecendo algumas recomendações. Eles ressaltam a necessidade de instituições e docentes reconhecerem e entenderem melhor os CoI para reduzir seus efeitos no planejamento, execução e publicação de pesquisas e na relação entre estudantes e professores, além de estabelecer regras que extrapolem a questão do financiamento de pesquisas ${ }^{66,79,80}$.

Com base no que foi apresentado até aqui, é possível listar diversos pontos a serem considerados no debate e na elaboração de medidas regulatórias da relação entre as indústrias de alimentos e as instituições que ofertam cursos de graduação em Nutrição, de forma a evitar: a participação de representantes das indústrias de alimentos e a utilização de material didático produzido por estas durante as disciplinas/módulos dos cursos; menção a marcas comerciais, produtos ou promoção de serviços específicos em sala de aula ou palestras voltadas a estudantes; distribuição de brindes, provenientes das indústrias de alimentos, a estudantes de nutrição; promoção ou distribuição de amostra grátis de fórmulas nutricionais infantis e de outros alimentos e produtos das indústrias de alimentos; patrocínio da indústria de alimentos em palestras/eventos; promoção das indústrias de alimentos em materiais educativos, livros didáticos, revistas científicas, entre outros; coleta de dados pessoais de estudantes para divulgação de produtos de indústrias alimentos por quaisquer vias (como carta, endereço eletrônico, redes sociais, entre outros); financia- mento, patrocínio ou doações de indústrias de alimentos para organizações/entidades estudantis; e o financiamento de indústrias de alimentos para instituições de ensino e pesquisa.

Ressalta-se ainda a necessidade de inclusão de conteúdos críticos em ementas de disciplinas e projetos pedagógicos de instituições de ensino sobre a atuação da indústria de alimentos para influenciar os profissionais de saúde; a promoção de debates sobre CoI com participação dos professores e pesquisadores; o desenvolvimento de guias e a criação de comitês que analisem os CoI; e a exigência de declaração de CoI entre palestrantes e indústrias de alimentos no início de aulas/palestras, caso ocorram.

Além do já discutido, cabe a reflexão sobre o papel do Estado na regulamentação dos CoI na formação de profissionais de saúde, principalmente no Brasil, onde o poder público possui grande importância como formador e empregador nesta área. A necessidade de se contrapor ao poder da indústria de alimentos, pela defesa do interesse público, precisa da atuação do Estado para estabelecer parâmetros e inibir ações danosas à formação dos nutricionistas.

\section{Considerações finais}

A partir do levantamento bibliográfico das estratégias utilizadas pelas indústrias de alimentos e de outras, cujas práticas são similares, para influenciar profissionais da área da saúde que trabalham com alimentação e nutrição e estudantes em vários espaços, verifica-se a necessidade de estabelecer regulamentos a fim de proteger, principalmente os estudantes, deste tipo de ação.

É responsabilidade do poder público, em conjunto com a sociedade civil, como universidades e estudantes, assumir o enfrentamento do debate sobre a regulação das estratégias utilizadas pelas indústrias de alimentos para influenciar profissionais da saúde e estudantes e garantir que a saúde pública seja priorizada em relação aos interesses privados ${ }^{16,17}$. Todavia, o processo de tomada de decisões sobre a regulação em diversos espaços não é acessível a todos os grupos de interesse e o envolvimento com a questão demanda tempo, recursos e exige comprometimento com a defesa e a participação em espaços de decisão política, os quais contam com participação ampla e ativa de lobistas, advogados e nutricionistas contratados pelas indústrias para defender seus interesses comerciais ${ }^{79}$, como mostram os exemplos e as pesquisas apresentados. 
No caso das universidades e faculdades, é necessário que sejam estabelecidos regulamentos ou códigos de ética relacionados aos CoI nos cursos da área da saúde, independentemente da normatização pelo Estado, podendo ter como base os pontos descritos neste artigo.

Além disso, professores dos cursos de Nutrição, nutricionistas e todos os demais envolvidos com a formação desse profissional devem agir com ética e promover debate com os estudantes e análise crítica das ações das indústrias, principalmente os grupos transnacionais, e suas diferentes formas de publicidade.

Ainda, devido à escassa literatura sobre o tema, é necessário desenvolver outros estudos que relacionem a área de Alimentação e Nutrição com os CoI na relação público-privado, desde a graduação até a atuação profissional, passando pela investigação desse tema em todo o ciclo de políticas públicas. A maioria dos materiais bibliográficos analisados se refere à análise da realidade nos Estados Unidos, assim, é necessária também a produção de trabalhos que avaliem diferentes realidades e estratégias que devem ser adotadas em cada local.

Essas ações são essenciais para que os estudantes recebam informações idôneas, provenientes de dados confiáveis e independentes de interesses comerciais, de forma que os nutricionistas formados possam desempenhar suas atribuições com ética e autonomia, promovendo melhores condições para a escolha de uma alimentação adequada e saudável, considerando que a alimentação humana não se relaciona apenas à necessidade biológica, mas se impregna pela cultura e pela sociedade que a cerca.

\section{Colaboradores}

TN Pereira, FA Nascimento e DH Bandoni participaram de todas as etapas de sua elaboração. 


\section{Referências}

1. Monteiro C, Cannon G, Claro R, Levy RB, Moubarac JC, Martins APB, Louzada ML, Baraldi L, Canella D. O sistema alimentar - O grande tema da nutrição: Uma nova classificação dos alimentos. São Paulo: NUPENS/ FSP/USP; 2012.

2. Connor JM, Schiek W. Food processing: an industrial powerhouse in transition. New York: John Wiley \& Sons; 1997.

3. Ludwig DS. Technology, diet, and the burden of chronic disease. JAMA 2011; 305(13):1352-1353.

4. Garcia RWD. Reflexos da globalização na cultura alimentar: considerações sobre as mudanças na alimentação urbana. Rev. Nutr. 2003; 16(4):483-492.

5. Belasco WJ. Appetite for change: How the counterculture took on the food industry. $3^{\text {rd }} \mathrm{ed}$. New York: Comstock Publishing Associates; 2007.

6. Moodie R, Stuckler D, Monteiro C, Sheron N, Neal B, Thamarangsi T, Lincoln P, Casswell S. Profits and pandemics: prevention of harmful effects of tobacco, alcohol, and ultra-processed food and drink industries. Lancet 2013; 381(9867):670-679.

7. Brasil. Ministério da Saúde (MS). Secretaria de Atenção à Saúde. Departamento de Atenção Básica. Coordenação-Geral de Alimentação e Nutrição. Guia Alimentar para a População Brasileira. Brasília: MS; 2014.

8. Monteiro CA, Castro IRR. Por que é necessário regulamentar a publicidade de alimentos. Ciênc. Cult. 2009; 61(4):56-59.

9. Figueiredo AV. A regulamentação de alimentos sob a ótica do Direito Sanitário. In: Brasil. Ministério da Saúde (MS). Secretaria de Atenção à Saúde. Departamento de Atenção Básica. Coordenação-Geral da Política de Alimentação e Nutrição. Seminário Nacional de Alimentação e Nutrição no SUS: PNAN 10 anos. Brasília: MS; 2010. [Relatório Final]. p. 20-23.

10. Monteiro CA. The underweight/overweight double burden for the poorest in low-income countries. In: Dube L, Bechara A, Dagher A, Drewnowski V, LeBel, James P, Yada RY, editors. Obesity prevention: the role of society and brain on individual behavior. New York: Elsevier; 2007. v.1. p. 463-469.

11. World Health Organization (WHO). Obesity: preventing and managing the global epidemic. Geneva: WHO; 2000. [Technical Report Series, 894].

12. Ferreira FR. Como pensar o capitalismo atual e suas consequências para o campo da nutrição sem recorrer à noção de estrutura? Demetra 2013; 8(Supl. 1):241245.

13. Pons SC. Pontos de Partida Teórico-metodológicos para o Estudo Sociocultural da Alimentação em um Contexto de Transformação. In: Canesqui AM, Garcia RWD, organizadores. Antropologia e Nutrição: Um diálogo possível. Rio de Janeiro: Fiocruz; 2005. p. 101-126.

14. Instituto Brasileiro de Geografia e Estatística (IBGE). Pesquisa Nacional de Saúde 2013: Ciclos de Vida Brasile Grandes Regiões. Rio de Janeiro: IBGE; 2015.

15. Swinburn BA, Sacks G, Hall KD, McPherson K, Finegood DT, Moodie ML, Gortmaker SL. The global obesity pandemic-shaped by global drivers and local environments. Lancet 2011; 378(9793):804-814.

16. Anon. The profession of nutrition: public-public partnerships. World Nutrition 2012; 3(11):476-482.
17. Brasil. Ministério da Saúde (MS). Secretaria de Atenção à Saúde. Departamento de Atenção Básica. Coordenação-Geral de Alimentação e Nutrição. Política Nacional de Alimentação e Nutrição. Brasília: MS; 2012. [Série B. Textos Básicos de Saúde]

18. Gomes FS, Castro IRR, Monteiro CA. Publicidade de alimentos no Brasil: avanços e desafios. Cienc. Cult. 2010. 62(4):48-51.

19. Witt JSGZ, Schnider AP. Nutrição e estética: valorização do corpo e da beleza através do cuidado nutricional. Cien Saude Colet 2011; 16(9):3909-3916.

20. Martins BR, Araújo IS, Jacob SC. A propaganda de alimentos: orientação ou apenas estímulo ao consumo? Cien Saude Colet 2011; 16(9):3873-3882.

21. Brownell KD, Warner KE. The Perils of Ignoring History: Big Tobacco Played Dirty and Millions Died. How Similar Is Big Food? Milbank Q 2009; 87(1):259-294.

22. Monteiro CA. The big issue is ultra-processing: there is no such thing as a health ultra-processed product. WPHNA [periódico na internet]. 2011 ago [acessado 2011 set 1]; 2(7). Disponível em: http://www.wphna. org/2011_aug_wn4_cam9.htm

23. Xavier C. Mídia e saúde, saúde na mídia. In: Santos A, organizador. Cad. mídia e saúde pública. Belo Horizonte: Funed; 2006. p. 43-55.

24. Nutrition Business Journal. NBJ's Global Supplement and Nutrition Industry Report 2014: an analisis of markets, trends, competition and strategy in the Global Nutrition Industry. Cleveland: Penton Media; 2014.

25. Spier R. Ethical issues in research relationships between universities and industry. Science and Engineering Ethics 1996; 2(1):115-120.

26. Brasil. Lei no 12.183 , de 16 de maio de 2013. Dispõe sobre o conflito de interesses no exercício de cargo ou emprego do Poder Executivo federal e impedimentos posteriores ao exercício do cargo ou emprego e dá outras providências. Diário Oficial da União 2013; 17 maio.

27. Freedhoff Y, Hébert PC. Partnerships between health organizations and the food industry risk derailing public health nutrition. CMAJ 2011; 183(3):291-292.

28. Nestle M. Food company sponsorship of nutrition research and Professional activities: a conflict of interest? PHN 2001; 4(5):1015-1022.

29. Rother ET. Revisão sistemática $X$ revisão narrativa. Acta Paul Enferm 2007; 20(2):5-6.

30. Deborah JC, Cynthia DM, Brian H. Systematic Reviews: Synthesis of Best Evidence for Clinical Decisions. Ann Intern Med 1997; 126(5):376-380.

31. World Health Organization (WHO). Creating an enabling environment for population-based salt reduction strategies. Geneva: WHO; 2010.

32. Nilson EAF, Jaime PC, Resende DO. Iniciativas desenvolvidas no Brasil para a redução do teor de sódio em alimentos processados. Rev. Panam Salud Publica 2012; 32(4):287-292.

33. Union of Concerned Scientists. Heads they win, tails we lose: how corporations corrupt science at the public's expense. Cambridge: UCS; 2012. [Report].

34. Jack A. Brazil unwanted growth. Financial Times [periódico na internet]. 2011 Apr 8 [acessado 2014 maio 29]. Disponível em: http://www.ft.com/intl/ cms/s/2/6e0319c2-5fee-11e0-a718-00144feab49a.ht$\mathrm{ml} \#$ axzz1lkKaaCdb 
35. Sobal J. Research ethics in nutrition education. J Nutr Educ 1992; 24(5):234-238.

36. Levine J, Gussow JD, Hastings D. Authors' Financial Relationships With the Food and Beverage Industry and Their Published Positions on the Fat Substitute Olestra. Am J Public Health 2003; 93(4):664-669.

37. Taubes G, Couzens CK. Big Sugar's Sweet Little Lies: How the industry kept scientists from asking: Does sugar kill? Mother Jones Nov/Dec 2012 Issue. [acessado 2013 jan 14]. http://www.motherjones.com/environment/2012/10/sugar-industry-lies-campaign

38. Wiley AS. "Drink Milk for Fitness": The Cultural Politics of Human Biological Variation and Milk Consumption in the United States. American anthropologist 2004; 106(3): 506-517.

39. Margetts B. Time to agree guidelines and apply an ethical framework for public health nutrition. PHN 2009; 12(7):885-886.

40. Castro IRR. World Nutrition Rio 2012. Rev Nutr 2011; 24(2):205-208.

41. Agência Nacional de Vigilância Sanitária. Resolução $\mathrm{n}^{\circ} 24$, de 2010. Dispõe sobre a oferta, propaganda, publicidade, informação e outras práticas correlatas cujo objetivo seja a divulgação e a promoção comercial de alimentos considerados com quantidades elevadas de açúcar, de gordura saturada, de gordura trans de sódio, e de bebidas com baixo teor nutricional. Diário Oficial da União 2010; 29 jun.

42. Henrique P, Dias PC, Burlandy L. A regulamentação da propaganda de alimentos no Brasil: convergências e conflitos de interesse. Cad Saude Publica 2014; 30(6):1219-1228.

43. Grynbaum MM. New York's ban on Big Sodas is rejected by Final Court. New York Times [periódico na internet]. 2014 Jun 26 [acessado 2014 ago 20]. Disponível em: http://www.nytimes.com/2014/06/27/nyregion/city-loses-final-appeal-on-limiting-sales-of-large-sodas. html?_r=0

44. Nixon R. New rules for school meals aim at reducing obesity. New York Times [periódico na internet]. 2012 Jan 25 [acessado 2014 jan 26]. Disponível em: http:// www.nytimes.com/2012/01/26/us/politics/new-school-lunch-rules-aimed-at-reducing-obesity.html? $\mathrm{r}=1 \&$ smid=tw-nytimes\&seid=auto

45. Yanamadala S, Bragg MA, Roberto CA. Food industry front groups and conflicts of interest: the case of Americans Against Food Taxes. PHN 2012; 15(8):1331-1332.

46. Soares JCRS, Deprá AS. Ligações perigosas: indústria farmacêutica, associações de pacientes e as batalhas judiciais por acesso a medicamentos. Physis 2012; 22(1):311-329.

47. Addams PJ. Assessing whether to receive funding support from tobacco, alcohol, gambling and other dangerous consumption industries. Addiction 2007; 102(7):1027-1033.

48. Waymack MH. Ethical conflicts of interest. JADA 2003; 103(5):555-557.

49. Gomes FS. Frutas, legumes e verduras: recomendações técnicas versus constructos sociais. Rev. Nutr. 2007; 20(6):669-680.
50. Brasil. Ministério da Saúde (MS). Secretaria de Atenção à Saúde. Departamento de Atenção Básica. Coordenação-Geral da Política de Alimentação e Nutrição. A iniciativa de incentivo ao consumo de Frutas, Verduras e Legumes (F,L\&V):uma estratégia intersetorial no contexto da Segurança Alimentar e Nutricional (CONSEA -Brasil). Brasília: MS; 2005. [Nota Técnica].

51. Brasil. Portaria GM nº154, de 24 de janeiro de 2008. Cria os Núcleos de Apoio à Saúde da Família. Diário Oficial da União 2008; 25 jan.

52. Brasil. Ministério da Saúde (MS). Secretaria de Atenção à Saúde. Departamento de Atenção Básica. Coordenação-Geral da Política de Alimentação e Nutrição. Matriz de ações de alimentação e nutrição na atenção básica de saúde. Brasília: MS; 2009. [Série A. Normas e Manuais Técnicos].

53. Daniel JMP, Cravo VZ. Valor Social e Cultural da Alimentação. In: Canesqui AM, Garcia RWD, organizadores. Antropologia e Nutrição: Um diálogo possível. Rio de Janeiro: Fiocruz; 2005. p. 57-68.

54. Conselho Federal de Nutrição. Resolução no 380, de 2005. Dispõe sobre a definição das áreas de atuação do nutricionista e suas atribuições, estabelece parâmetros numéricos de referência, por área de atuação, e dá outras providências. Diário Oficial da União 2006; 10 jan.

55. Brasil. Ministério da Educação (ME). Parecer CNE/ CES no 1133, de 1 de outubro de 2001. Diretrizes curriculares nacionais dos cursos de graduação em Enfermagem, Medicina e Nutrição. Diário Oficial da União 2001; 3 out.

56. Beauman C, Cannon G, Elmadfa I, Glasauer P, Hoffmann I, Keller M, Krawinkel M, Lang T, Leitzmann C, Lötsch B, Margetts BM, McMichael AJ, Meyer-Abich K, Oltersdorf U, Pettoello-Mantovani M, Sabaté J, Shetty P, Sória M, Spiekermann U, Tudge C, Vorster HH, Wahlqvist M, Zerilli-Marimò M. The principles, definition and dimensions of the new nutrition science. Public Health Nutr 2005; 8(6A):695-698.

57. Canesqui AM, Garcia RWD. Ciências Sociais e Humanas nos Cursos de Nutrição. In: Canesqui AM, Garcia RWD, organizadores. Antropologia e Nutrição: Um diálogo possivel. Rio de Janeiro: Fiocruz; 2005. p. 255-274.

58. Simon M. And now a word from our sponsors: Are America's Nutrition professionals in the pocket of Big Food? Eat Drink Politics 2013. [Report].

59. Hampl JS, Anderson JV, Mullis R, American Dietetic Association. Position of the ADA: the role of Dietetics Professionals in Health Promotion and Disease Prevention. JADA 2002; 102(11):1680-1687.

60. Reitshamer E, Schrier MS, Herbold N, Metallinos-Katsaras E. Members' Attitudes Toward Corporate Sponsorship of the Academy of Nutrition and Dietetics. J Hunger Environ Nutr 2012; 7(2-3):149-164.

61. Trevisol DJ, Ferreira MBC, Karnopp ZMP. A propaganda de medicamentos em escola de medicina do Sul do Brasil. Cien Saude Colet 2010; 15(Supl. 3):3487-3496.

62. Palacios M, Rego S, Lino MH. Promoção e propaganda de medicamentos em ambientes de ensino: elementos para o debate. Interface (Botucatu) 2008; 12(27):893905. 
63. Rea MF, Toma TS. Proteção do leite materno e ética. Rev Saude Publica 2000; 34(4):388-395.

64. Fagundes MJD, Soares MGA, Diniz NM, Pires JR, Garrafa V. Análise bioética da propaganda e publicidade de medicamentos. Cien Saude Colet 2007; 12(1):221-229.

65. MacDonald C, Williams-Jones B. Supervisor-Student Relations: Examining the Spectrum of Conflicts of Interest in Bioscience Laboratories. Account Res. 2009; 16(2):106-126

66. Silva FL. Reflexões sobre o conceito e a função da universidade pública. Estudos Avançados 2001; 15(42):295304.

67. Capella ACN. Perspectivas Teóricas sobre o Processo de Formulação de Políticas Públicas. BIB 2006; 61:25-52.

68. Natale SM, Doran C. Marketization of Education: An Ethical Dilemma. J Bus Ethics 2012; 105(2):187-196.

69. Wofford JL, Ohl CA. Teaching appropriate interactions with pharmaceutical company representatives: The impact or an innovative workshop on student attitudes. BMC Med. Educ. 2005 [periódico na internet]. 5(5). [acessado 25 maio 2011]. Disponível em: http://www. biomedcentral.com/1472-6920/5/5

70. Wazana A. Physicians and the pharmaceutical industry: is a gift ever just a gift? JAMA 2000; 283(3):373-380.

71. Mccormick BB, Tomlinson G, Brill-Edwards P, Detsky AS. Effect of restricting contact between pharmaceutical company representatives and internal medicine on posttraining attitudes and behavior. JAMA 2001. 286(16):1994-1999.

72. Rogers WA, Mansfield PR, Braunack-Mayer AJ, Jureidini JN. The ethics of pharmaceutical industry relationships with medical students. MJA 2004; 180(8):411414.

73. Kao AC, Braddock C 3rd, Clay M, Elliott D, Epstein SK, Filstead W, Hotze T, May W, Reenan J. Effect of Educational Interventions and Medical School Policies on Medical Students' Attitudes Toward Pharmaceutical Marketing Practices: A Multi-Institutional Study. Acad Med 2011; 86(11):1454-1462.
74. Dally M. Ethical considerations raised by the provision of freebies to veterinary students. J Am Vet Med Assoc 2011; 238 (12):1551-1554.

75. Veloso TCMA, Souza BKG, Silva, RAB. Cursos de graduação em nutrição no Brasil: análise do censo da educação superior do Brasil e os resultados do Enade. Educação e Fronteiras On-Line 2011; 1(1):92-106.

76. Vasconcelos FAG, Calado CLA. Profissão nutricionista: 70 anos de história no Brasil. Rev. Nutr. 2011; 24(4):605-617.

77. Zipkin DA, Steinman MA. Interactions between pharmaceutical representatives and doctors in training: a thematic review. J Gen Intern Med 2005; 20(8):777-786.

78. Australian Medical Students Association (AMSA). Guidelines for the interaction between pharmaceutical companies and Australian medical students. Sidney: AMSA; s.d.

79. Kellogg S. The power of transparency. Nature 2012; (484):131-133.

80. Lawrence M. Do food regulatory systems protect public health? PHN 2009; 12(11):2247-2249.

Artigo apresentado em 07/11/2014

Aprovado em 03/09/2015

Versão final apresentada em 05/09/2015 